\title{
Electromagnetic Signal Propagation in a Lorentz Dispersive Medium
}

\author{
Adam Ciarkowski
}

\begin{abstract}
This work is concerned with the propagation of rapidly oscillating electromagnetic (EM) signal in a Lorentz dispersive medium. The problem considered here is 1-dimensional and its exact solution is described by a contour integral defined in a complex frequency plane. With the use of uniform asymptotic techniques, approximate representation for the total field consisting of the Sommerfeld and Brillouin precursors and the main signal is obtained. The effect of the rate of envelope changes, as well as of carrier frequency on the shape of the total signal is examined.
\end{abstract}

Keywords-Dispersion, Sommerfeld precursor, Brillouin precursor.

\section{INTRODUCTION}

A Temporal dispersive medium is the medium whose refraction index $n=\sqrt{\epsilon \mu}$ is frequency dependent. Responsible for this feature is the polarization phenomenon, induced in the medium by time varying, exterior electric field. As a result, the relative electric permittivity $\epsilon$ varies with frequency. Magnetic properties of the medium do not interfere, and hence the relative magnetic permeability $\mu$ may be considered constant.

Except for the special medium - vacuum, all remaining media are dispersive. When studying electromagnetic phenomena usually we do not take dispersion into account. At relatively low frequencies such an assumption is fully justified, since polarization variations exactly follow electric forces induced by the varying electric field. At higher frequencies, however, this process is more complex, because of inertia of dipoles in the medium (see [1]). A widely used model for a dispersive medium has been proposed by Lorentz. Its complex refraction index takes the form:

$$
n(\omega)=\left(1-\frac{b^{2}}{\omega^{2}-\omega_{0}^{2}+2 i \delta \omega}\right)^{1 / 2} .
$$

Here, $b^{2}=4 \pi N e^{2} / m$ is the plasma frequency of the medium, $N, e$ and $m$ are the number of electrons per unit volume, electron charge and its mass, respectively, $\delta$ is a damping constant and $\omega_{0}$ is the resonant frequency (comp. [1], Sec.7.10). The real and imaginary parts of the complex index of refraction of a dispersive medium with Brillouin choice of medium parameters: $\omega_{0}=4.0 \times 10^{1} 6 s^{-1}, b=\sqrt{20.0} \times 10^{1} 6 s^{-1}$, $\delta=0.28 \times 10^{1} 6 s^{-1}$ is shown in Fig. 1 .

This work was partially supported by Polish Ministry of Education and Science with funds granted for research in 2011-2013 (Grant No. O N517 418440).

A. Ciarkowski is with the Warsaw University of Life Sciences, Faculty of Applied Informatics and Mathematics, Nowoursynowska 159, 02-776 Warsaw, Poland (e-mail: adam_ciarkowski@sggw.pl).

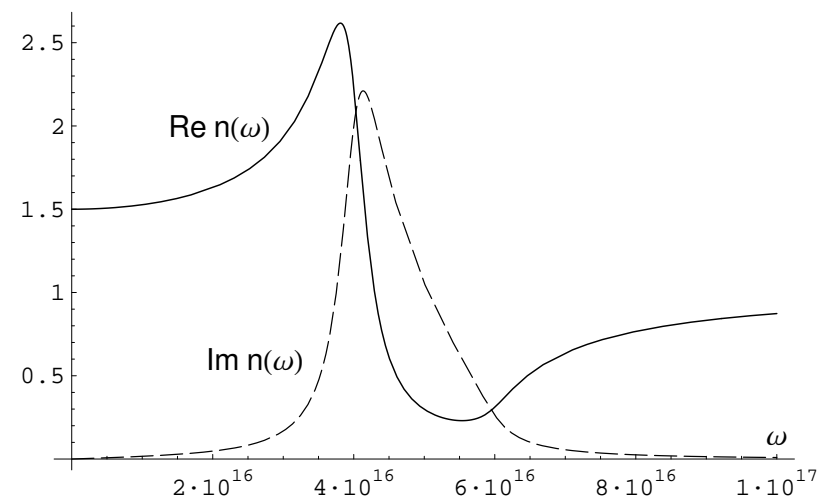

Fig. 1. Real and imaginary parts of the complex index of refraction of an exemplary dispersive medium as functions of angular frequency $\omega$ for Brillouin choice of medium parameters: $\omega_{0}=4.0 \times 10^{16} s^{-1}, b=\sqrt{20.0} \times$ $10^{16} s^{-1}, \delta=0.28 \times 10^{16} s^{-1}$.

The beginnings of the analysis of electromagnetic propagation in dispersive media date back to the beginning of the twentieth century. Fundamental works on EM signals propagation in a Lorentz medium are due to Sommerfeld [2] and Brillouin [3], [4]. The authors showed that in addition to the main signal propagating in the medium, two precursors are formed that precede the signal. The front of the fastest (Sommerfeld) precursor propagates in the medium with the velocity of light. The instantaneous oscillation frequency of the precursors and their local damping are directly related to the locations in the complex frequency plane of corresponding saddle points in the integral description of the signal. Those locations vary with space and time, and are governed by the saddle point equation, requiring that the phase function in the integrand be stationary. Analysis of that equation showed that there are two pairs of dominant saddle points, distant and near ones, responsible for the first (referred to as Sommerfeld) and the second (Brillouin) precursors, respectively.

Initially, the analysis of the integral solution for the dispersive field was rather troublesome, due to the fact that the integrand variations are very fast, and at that time there was a lack of efficient examination methods. After asymptotic techniques were developed, a powerful analytical tool got feasible, and the study of a signal evolution in a dispersive medium experienced significant acceleration. One of the advantages of the asymptotic approach is that the problem has simplified, but still analytic representation. Moreover, each physical signal, irrespectively of its magnitude, has its own mathematical representation, and therefore its behavior may be analyzed as its different parameters are varied. Such an analysis has a practical meaning. E.g. in order to be able 
to differentiate between the head of the main signal and the heads of its precursors on EM signal arrival, the form of the composite signal should be known.

Usually solutions obtained with the use of simple asymptotic methods are sufficiently accurate for different values of the parameters. However, for some parameter configurations they break down. This happens when corresponding different "critical" points coalesce on each other. To overcome this inconvenience "uniform" asymptotic methods were introduced, as extensions of the "non-uniform" ones. (comp. [5], [6]). In the present context, the uniform asymptotic approach allows for full description of the head of the Sommerfeld precursor, evolution of the Brillouin precursor, and smooth transition from that precursor to the main signal.

The issues of uniqueness, causality and regularity of a pulse propagating in a dispersive, nonuniform medium are discussed in [7]. Detailed analysis of dispersive propagation is given in two monographs, [8] and [9]. The former deals with dispersive processes in fluids, the latter - with electomagnetic propagation. Still however, some problems given in [9] and later publications remain controversial, and are subject of critical discusion (see e.g. [10] and [11]). Other recent results on precursors in dispersive media can be found in [12], [13] and [14]. One of the inconsistences in [9] is that the representation of the Sommerfeld precursor at its front does not follow the behavior predicted by Sommerfeld. Here, a different precursor representation is presented. Our result fully agrees with the Sommerfeld prediction. Also the transition from the Brillouin precursor to the main signal is here treated in a different manner. We take advantage of earlier works, [15], [16], [17] and [18] to collect the particular component fields into a compound signal. The dynamics of that signal is presented for different values of the parameters.

\section{Problem Formulation and Its Exact Solution}

Assume that at the moment $t=0$ the signal is produced in the plane $z=0$. Because the considered 1-dimensional problem depends on $z$-coordinate only, the field is uniform in all directions perpendicular to the $z$-axis. It is described by a harmonic signal, modulated by the function with finite rise-time

$$
A(0, t)= \begin{cases}0 & t<0 \\ \tanh (\beta t) \sin \left(\omega_{c} t\right) & t \geq 0 .\end{cases}
$$

The function $A(0, t)$ can be any coordinate of the electric or magnetic field, or the Lorentz potential. Large positive coefficient $\beta$ determines how rapidly the signal turns on, and $\omega_{c}$ is a carrier frequency. It is also assumed that no EM sources are present at $z \rightarrow \infty$.

In general, the problem consists in finding the field in the half-space $z>0$ and time $t>0$. The solution to this mixed initial-boundary value problem for Maxwell equations takes the form [15]

$$
A(z, t)=\frac{1}{2 \pi} \int_{i a-\infty}^{i a+\infty} g\left(\omega ; \beta, \omega_{c}\right) \exp \left[i \frac{z}{c} \Psi(\omega, \theta)\right] d \omega,
$$

where the constant $a$ is greater than the abscissa of absolute convergence for $A(0, t)$. The amplitude and phase functions $g\left(\omega ; \beta, \omega_{c}\right)$ and $\Psi(\omega, \theta)$, respectively, are given by

$$
\begin{aligned}
& g\left(\omega ; \beta, \omega_{c}\right)= \\
& \frac{1}{2}\left\{\frac{i}{\beta} \mathcal{B}\left[-\frac{i\left(\omega-\omega_{c}\right)}{2 \beta}\right]+\frac{1}{\omega-\omega_{c}}\right. \\
&\left.\quad-\frac{i}{\beta} \mathcal{B}\left[-\frac{i\left(\omega+\omega_{c}\right)}{2 \beta}\right]-\frac{1}{\omega+\omega_{c}}\right\}
\end{aligned}
$$

and

$$
\Psi(\omega, \theta)=\omega[n(\omega)-\theta]
$$

The beta function is defined by the psi function as

$$
\mathcal{B}(s)=\frac{1}{2}\left[\psi\left(\frac{s+1}{2}\right)-\psi\left(\frac{s}{2}\right)\right] .
$$

(For the definition and properties of the psi function see [19], Sec. 6.3.) The beta function is related to the envelope of $A(0, t)$ via the Fourier transformation

$$
\int_{0}^{\infty} \tanh \beta t e^{i \omega t} d t=\frac{1}{\beta} \mathcal{B}\left(-\frac{i \omega}{2 \beta}\right)-\frac{i}{\omega} .
$$

Finally,

$$
\theta=\frac{c t}{z}
$$

is a dimensionless parameter that characterizes a space-time point $(z, t)$.

\section{Asymptotic Solution}

The uniform asymptotic representation for the Sommerfeld precursor is given by ([15])

$$
\begin{aligned}
& A^{S}(z, t) \sim-\exp \left\{-\lambda \operatorname{Im}\left[\psi\left(\omega_{s}^{+}, \theta\right)\right]\right\} \\
& \times\left\{\operatorname{Re}\left[G\left(\omega_{s}^{+} ; \beta, \omega_{c}\right)\right] J_{1}\left[-\lambda \operatorname{Re}\left(\psi\left(\omega_{s}^{+}, \theta\right)\right)\right]+\right. \\
& \left.\quad \operatorname{Im}\left[G\left(\omega_{s}^{+} ; \beta, \omega_{c}\right)\right] J_{2}\left[-\lambda \operatorname{Re}\left(\psi\left(\omega_{s}^{+}, \theta\right)\right)\right]\right\},
\end{aligned}
$$

where

$$
\begin{gathered}
G\left(\omega_{s}^{+} ; \beta, \omega_{c}\right)=\sqrt{\frac{\operatorname{Re}\left[\psi\left(\omega_{s}^{+}, \theta\right)\right]}{\psi_{\omega \omega}\left(\omega_{s}^{+}, \theta\right)}} g\left(\omega_{s}^{+} ; \beta, \omega_{c}\right), \\
\lambda=z / c,
\end{gathered}
$$

and $J_{1}(\cdot)$ and $J_{2}(\cdot)$ are Bessel functions of the order 1 and 2, respectively.

The pair of distant simple saddle points, $\omega_{s}^{+}(\theta)$ and $\omega_{s}^{-}(\theta)$, are varying in the complex frequency plane symmetrically with respect to the imaginary axis. Their location is governed by the saddle point equation (see [15])

$$
n(\omega)+\omega n^{\prime}(\omega)-\theta=0 .
$$

Let us examine the precursor dependence on the speed parameter $\beta$. If $\beta$ is steadily increased starting from its relatively small values, the oscillations in Fig. 2 are increased by the same factor, while the shape of the precursor is preserved. If, however, $\beta$ takes much higher values, such as $\beta=1.0 \times 10^{19} s^{-1}$ or more, the precursor shape is clearly changed (see Fig. 3), and the oscillation amplitudes virtually remain at the same relatively high level as $\beta$ further increases.

It is seen from Fig. 4 that there are three characteristic regions of $G\left(\omega_{s}^{+} ; \beta, \omega_{c}\right)$ variation. If for fixed $\omega_{s}^{+}$the parameter 


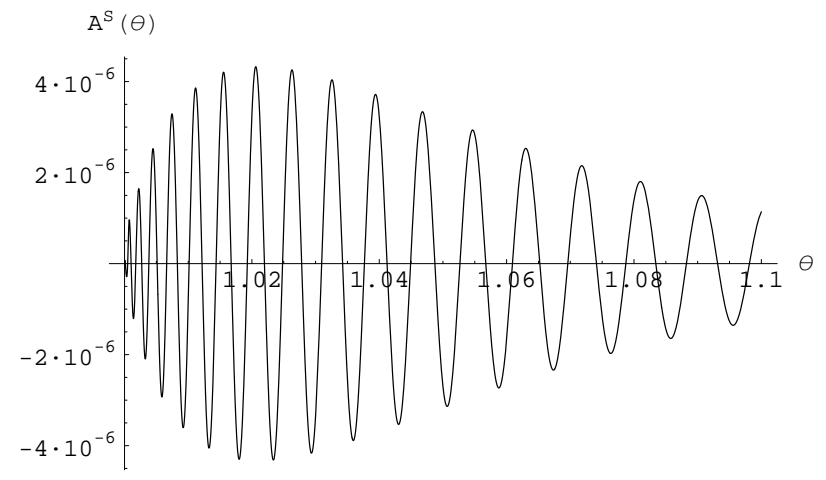

Fig. 2. Dynamic behavior of the Sommerfeld precursor in the Lorentz medium described by Brillouin choice of parameters. Here: $\omega_{c}=2.0 \times$ $10^{16} s^{-1}, \lambda=5.0 \times 10^{-15} s$ and $\beta=1.0 \times 10^{14} s^{-1}$.

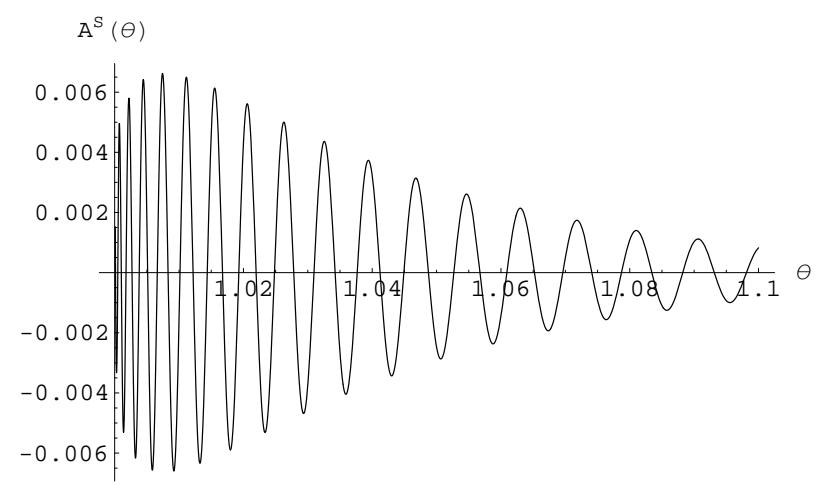

Fig. 3. Dynamic behavior of the Sommerfeld precursor in the Lorentz medium described by Brillouin choice of parameters. Here: $\omega_{c}=2.0 \times$ $10^{16} s^{-1}, \lambda=5.0 \times 10^{-15} s$ and $\beta=1.0 \times 10^{19} s^{-1}$.

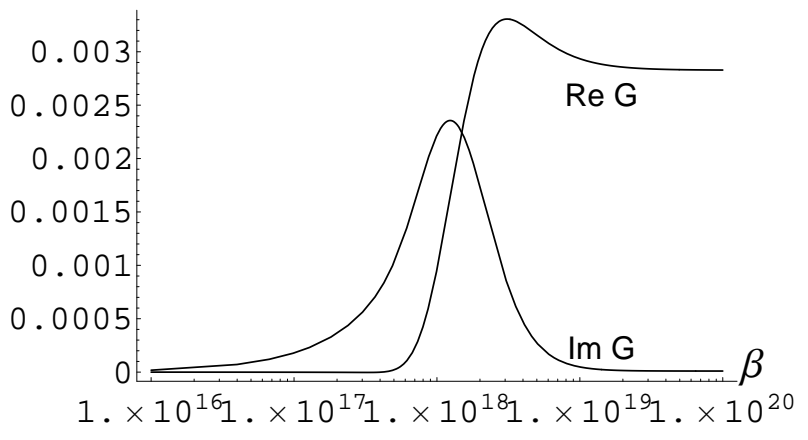

Fig. 4. Dependence of the real and imaginary parts of the function $G\left(\omega_{s}^{+} ; \beta, \omega_{c}\right)$ on the parameter $\beta$. Calculated for $\theta=1.0001$ and $\omega_{c}=$ $2.0 \times 10^{16} s^{-1}$ $\beta$ is relatively small then the real part of function $G\left(\omega_{s}^{+} ; \beta, \omega_{c}\right)$ is virtually zero and the essential contribution to the precursor is due to its imaginary part. This contribution increases in value with rising $\beta$ until about $\beta=4.0 \times 10^{17} \mathrm{~s}^{-1}$. This is the first region in which the precursor oscillation is governed by the Bessel function $J_{2}$. In the second, transitory region, the real part of $G$ grows rapidly, and at about $\beta=2.0 \times 10^{19} \mathrm{~s}^{-1}$ it settles down at a virtually constant level. The imaginary part reaches its maximum and then steadily decreases to zero. Here the Bessel function $J_{1}$ takes over. Finally, in the third region the real part of $G$ remains nearly unchanged and the imaginary part vanishes. Now $J_{1}$ dominates and that of $J_{2}$ is to be neglected. One can verify that with increasing $\theta$, the third region broadens, thus pushing the second region in the direction of smaller $\beta$.

\section{A. Special Case}

Assume that $\beta \rightarrow \infty$ and $\theta \simeq 1^{+}$. Then $g\left(\omega_{s}^{+} ; \beta, \omega_{c}\right) \approx$ $-2 \omega_{c} \sqrt{2(\theta-1)} b^{-2}$, and (9) reduces to

$$
A^{S}(z, t) \approx \frac{\omega_{c} \sqrt{2(\theta-1)}}{b} J_{1}(\lambda b \sqrt{2(\theta-1)}) .
$$

It is readily seen that this result fully agrees with the representation

$$
A^{S}(z, t) \approx \frac{2 \pi}{\tau} \sqrt{\frac{\mathfrak{t}}{\xi}} J_{1}(2 \sqrt{\mathfrak{t} \xi}),
$$

obtained by Sommerfeld on the grounds of integral considerations and valid for the initial signal described by the Heaviside unit step function ([4], Eq. (33)). Here, we have employed Sommerfeld's notation:

$$
\mathfrak{t}=t-\frac{z}{c} \quad \xi=\frac{b^{2} z}{2 c} \quad \tau=\frac{2 \pi}{\omega_{c}} .
$$

One can note that the asymptotic representation found in [9] does not agree with (13).

We now turn to the Brillouin precursor. Its behavior is dependent on the dynamics of a different pair of saddle points, also being subject to the equation (12). As $\theta$ is increased from the value 1 , these points, to be denoted by $\omega_{b}^{+}$and $\omega_{b}^{-}$, approach each other along the imaginary $\omega$-axis from below and from above, respectively. At some $\theta=\theta_{s}$ they coalesce at a point on the axis, thus forming one saddle point of the second order. As $\theta$ is further increased they depart from the axis and move symmetrically in the right and left $\omega$ half-planes, respectively. The asymptotic representation for the Brillouin precursor is given by (see [18])

$$
\begin{aligned}
A^{B}(z, t) \sim 2 \pi i e^{\lambda \rho_{b}(\theta)}( & \frac{c_{0}(\theta)}{\lambda^{1 / 3}} A i\left[\lambda^{2 / 3} \gamma_{b}(\theta)^{2}\right] \\
& \left.+\frac{c_{1}(\theta)}{\lambda^{2 / 3}} A i^{\prime}\left[\lambda^{2 / 3} \gamma_{b}(\theta)^{2}\right]\right),
\end{aligned}
$$

where

$$
\rho_{b}=\frac{1}{2}\left[\Psi\left(\omega_{b}^{+}\right)-\Psi\left(\omega_{b}^{-}\right)\right],
$$

and

$$
\gamma_{b}=\left[\frac{3}{4}\left|\phi\left(\omega_{b}^{+}\right)-\phi\left(\omega_{b}^{-}\right)\right|\right]^{1 / 3} e^{i \alpha},
$$




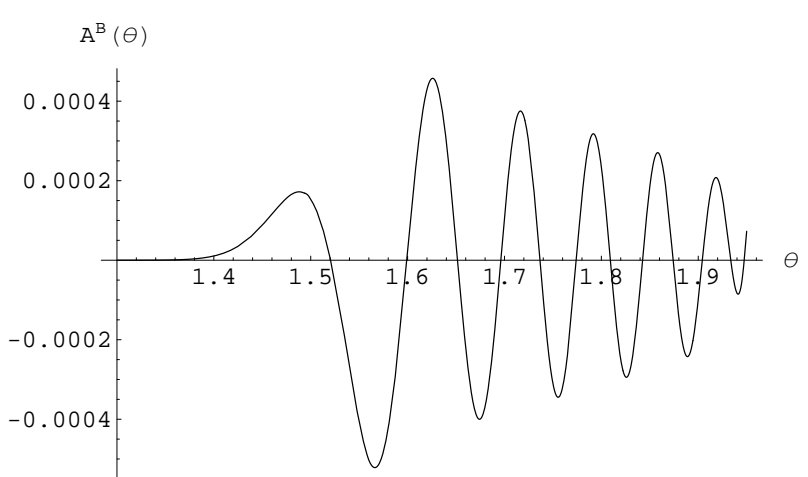

Fig. 5. Dynamic behavior of the Brillouin precursor in the Lorentz medium described by Brillouin choice of parameters. Here: $\omega_{c}=2.0 \times 10^{16} s^{-1}$ $\lambda=5.0 \times 10^{-15} s$ and $\beta=1.0 \times 10^{14} s^{-1}$

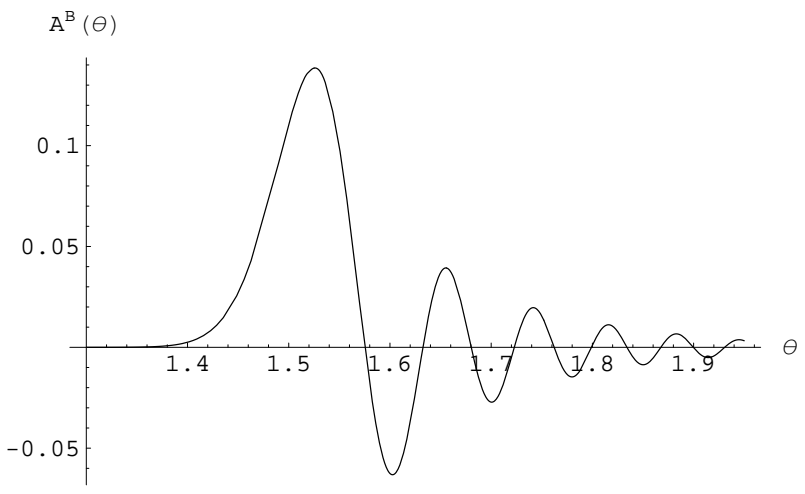

Fig. 6. Dynamic behavior of the Brillouin precursor in the Lorentz medium described by Brillouin choice of parameters. Here: $\omega_{c}=2.0 \times 10^{16} s^{-1}$, $\lambda=5.0 \times 10^{-15} s$ and $\beta=1.0 \times 10^{19} s^{-1}$.

with $\alpha=0,-\pi / 2$ if $\theta<\theta_{s}$ or $\theta>\theta_{s}$, respectively. The expansion is described by the Airy function and its derivative. It remains valid for any $\gamma_{b}(\theta)$, including $\gamma_{b}=0$, where the simple saddle points coalesce into one saddle point of the second order. Thus the expansion is uniform in $\gamma_{b}$, and hence in $\theta$.

The dependence of the Brillouin precursor on $\beta$ is similar as in the Sommerfeld precursor. As before, for relatively small values of this parameter the magnitude of the precursor is proportional

to $\beta$. With growing $\beta$ the precursor magnitude reaches a saturation level and further increase of $\beta$ practically does not change the precursor magnitude. Exemplary forms of the Brillouin precursor for $\beta$ differing by three orders are shown in Fig. 5 and Fig. 6.

Explanation for this precursor behavior is similar as in the previous case. $\mathrm{A}$ is seen from Fig. 7, at lower values of $\beta$ the coefficient $c_{1}$ dominates over $c_{0}$ and hence the precursor behavior is described by the derivative of the Airy function. At large values of $\beta$, the coefficient $c_{0}$ prevails, and the dynamics of the precursor is described by the Airy function itself.

The final signal component is the main signal. Essential in obtaining the smooth transition from Brillouin precursor to the main signal is a proper handling of the case in which the

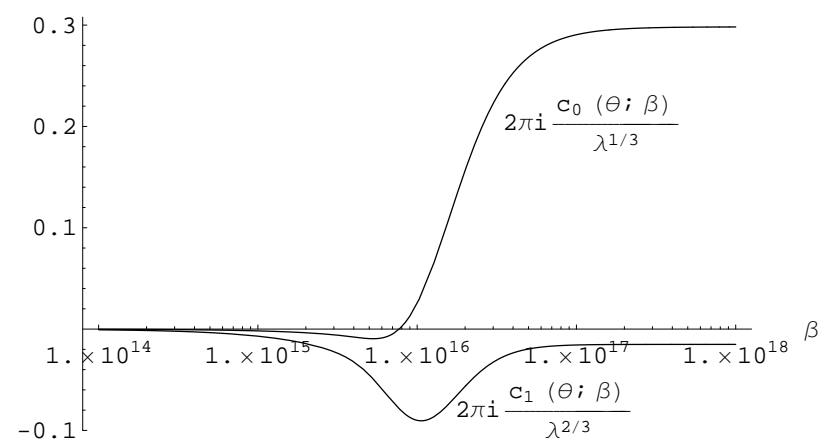

Fig. 7. Plots of $2 \pi i \lambda^{-1 / 3} c_{0}(\theta)$ and $2 \pi i \lambda^{-2 / 3} c_{1}(\theta)$ against the speed parameter $\beta$ at $\theta=1.502$. Here, $\omega_{c}=2.5 \times 10^{16} s^{-1}$ and $\lambda=3.0 \times 10^{-15} s$.

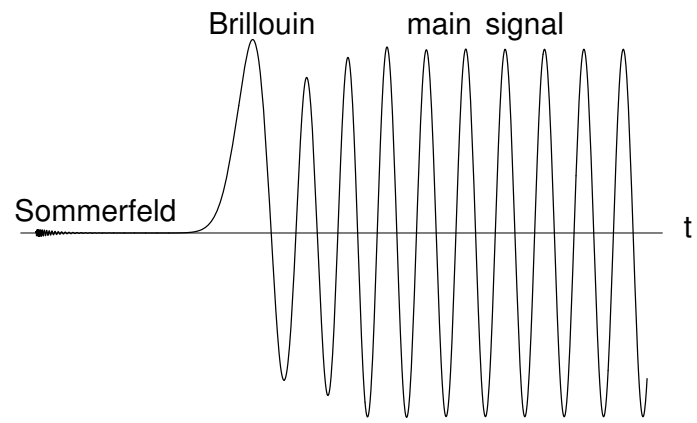

$\omega_{\mathrm{c}}=1.310^{16} \mathrm{~s}^{-1}$

Fig. 8. Dynamic behavior of the total dispersive signal in the Lorentz medium described by Brillouin choice of parameters. Here: $\omega_{c}=1.3 \times 10^{16} s^{-1}$, $\lambda=5.0 \times 10^{-15} s$ and $\beta=1.0 \times 10^{19} s^{-1}$.

deformed contour of integration crosses the pole at $\omega=\omega_{c}$, and either saddle point $\omega_{s p}=\omega_{b}^{+}$or $\omega_{s p}=\omega_{s}^{+}$approaches the pole. Resulting formula for the main signal contribution is given by (see [17])

$$
\begin{aligned}
& A_{m}(z, t) \sim \operatorname{Re}\left\{e ^ { \lambda \rho _ { m } } \left[\frac{i}{2} \operatorname{erfc}\left(i \gamma_{m} \sqrt{\frac{\lambda}{2}}\right)\right.\right. \\
& \left.\left.-\frac{e^{\frac{\lambda \gamma_{m}^{2}}{2}}}{\sqrt{2 \pi \lambda}}\left(\frac{1}{\gamma_{m}}+\frac{1}{\left(\omega_{s p}-\omega_{c}\right) \sqrt{-\Psi_{\omega \omega}\left(\omega_{s p}, \theta\right)}}\right)\right]\right\},
\end{aligned}
$$

Here,

$$
\rho_{m}=\Psi\left(\omega_{c}, \theta\right), \quad \gamma_{m}=\sqrt{2\left[\Psi\left(\omega_{s p}, \theta\right)-\Psi\left(\omega_{c}, \theta\right)\right.},
$$

and erfc stands for the complementary error function.

In Fig. 8 through Fig. 10 the full dispersive signal is shown, as found for different carrier frequency $\omega_{c}$ in the main signal. It is seen that when approaching the frequency region of anomalous dispersion the main signal is attenuated, an at the same time the precursors contribution is enlarged.

\section{CONCLUSiOnS}

The effect of dispersion on signal propagation in a medium is shown. In a mature dispersive regime, in addition to the main signal two precursors are generated. Their magnitudes strongly depends on the rate at which the envelope of the 


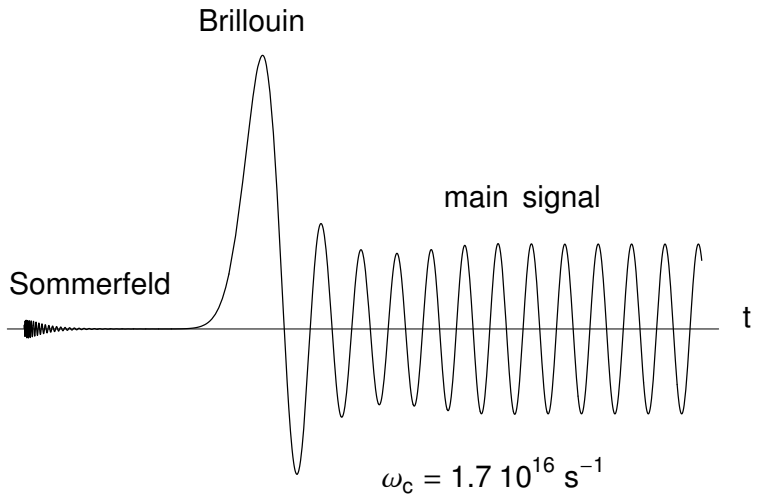

Fig. 9. Dynamic behavior of the total dispersive signal in the Lorentz medium described by Brillouin choice of parameters. Here: $\omega_{c}=1.7 \times 10^{16} s^{-1}$, $\lambda=5.0 \times 10^{-15} s$ and $\beta=1.0 \times 10^{19} s^{-1}$

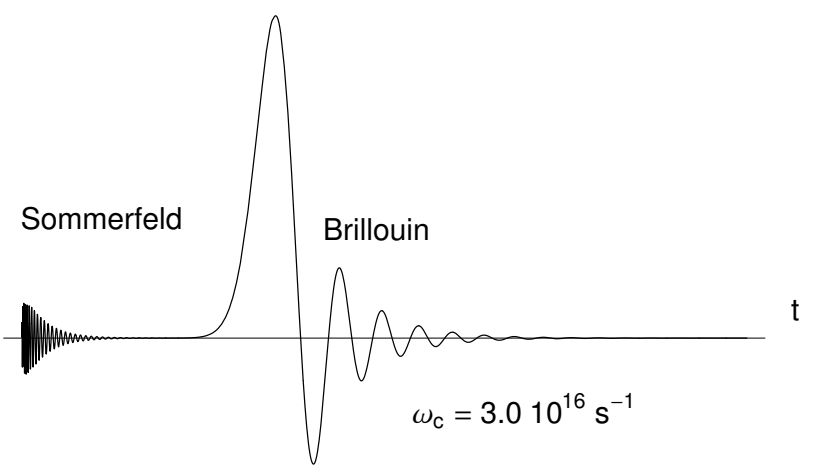

Fig. 10. Dynamic behavior of the total dispersive signal in the Lorentz medium described by Brillouin choice of parameters. Here: $\omega_{c}=3.0 \times$ $10^{16} s^{-1}, \lambda=5.0 \times 10^{-15} s$ and $\beta=1.0 \times 10^{19} s^{-1}$.

main signal changes. Their relative contents also vary with the carrier frequency of the main signal.

Smooth representation of the total signal was obtained with the help of uniform asymptotic techniques. In case of simpler, non-uniform techniques it is impossible to properly represent the head of the Sommerfeld precursor, transition from the increasing to the oscillation part in the Brillouin precursor and the smooth transition from that precursor to the main signal.

\section{REFERENCES}

[1] J. D. Jackson, Classical Electrodynamics, 3rd ed. John Wiley, 1999.

[2] A. Sommerfeld, "Über die fortpflanzung des lichtes in dispergieren den medien," Annalen der Physik, vol. 44, 1914.

[3] L. Brillouin, "Über die fortpflanzung des lichtes in dispergieren den medien," Annalen der Physik, vol. 44, 1914.

[4] - Wave Propagation and Group Velocity. Academic Press, 1960.

[5] N. Bleistein and R. A. Handelsman, Asymptotic Expansions of Integrals. Holt, Rinehart and Winston, 1975

[6] L. B. Felsen and N. Marcuvitz, Radiation and Scattering of Waves. Prentice-Hall, 1974

[7] S. Rikte, "Existence, uniqueness, and causality theorems for wave praopagation in stratiffied, temporally dispersive, complex media," SIAM Journal on Applied Mathematics, vol. 57, 1997.

[8] M. Kelbert and I. Sazonov, Pulses and Other Wave Processes in Fluids. Kluwer, 1996

[9] K. E. Oughstun and G. C. Sherman, Electromagnetic Pulse Propagation in Causal Dielectrics. Springer, 1997.

[10] K. E. Oughstun, N. A. Cartwright, D. J. Gauthier, and H. Jeong, "Optica precursors in the singular and weak dispersion limits," Journal of Optical Society of America, vol. B 27, 2010.

[11] B. Macke and B. Ségard, "Comment on iioptical precursors in the singular and weak dispersion limits $i i, " J o u r n a l$ of Optical Society of America, vol. B 28, 2011.

[12] _ , "Optical precursors in transparent media," Physical Review, vol. A : Atomic, Molecular and Optical Physics, 2009.

[13] J. F. Chen, M. M. T. Loy, G. K. L. Wong, and S. Du, "Optical precursors with finite rise and fall time," Journal of Optics, vol. 12, 2010

[14] C. F. Li and G. C. G. Z. Q. Zhou, H. Jeong. (2011, Feb.) Directly observable speed of optical precursors. pdf:1102.4998v1. [Online] Available: arxiv.org

[15] A. Ciarkowski, "On sommerfeld precursor in a lorentz medium," Journal of Technical Physics, vol. 43, 2002.

[16] —, "Approximate representation for the brillouin precursor in a lorentz medium," Kwartalnik Elektroniki i Telekomunikacji, vol. 48, 2002.

[17] _ "Propagation of the main signal in a dispersive lorentz medium," Archives of Acoustics, vol. 27, 2002.

[18] _ _ "Dependence of the brillouin precursor form on the initial signa rise time," Journal of Technical Physics, vol. 44, 2003.

[19] M. Abramowitz and I. Stegun, Handbook of Mathematical Functions. National Bureau of Standards, Applied Mathematics, 1964. 